\title{
Transcaval transcatheter aortic valve replacement: a visual case review
}

\author{
Kamran I. Muhammad, Georgianne C. Tokarchik \\ Oklahoma Heart Institute, Tulsa, Oklahoma, USA \\ Correspondence to: Kamran I. Muhammad. Oklahoma Heart Institute, 1265 South Utica Suite 300, Tulsa, Oklahoma, 74104, USA. \\ Email: kamran.muhammad@oklahomaheart.com.
}

\begin{abstract}
Transcatheter aortic valve replacement (TAVR) has emerged as a viable, minimally-invasive and widely adopted approach for the treatment of severe symptomatic aortic stenosis in patients who are intermediate-risk or greater for surgical aortic valve replacement. Numerous studies have demonstrated favorable outcomes with TAVR in this population, particularly with transfemoral access TAVR. Transfemoral TAVR has been shown to be safer and associated less morbidity, shorter lengths of hospital stay and more rapid recovery as compared with traditional thoracic alternative-access TAVR (transapical or transaortic). Despite iterative advancements in transcatheter heart valve technology and delivery system, there remain a portion of patients with iliofemoral arterial vessel sizes that are too small for safe transfemoral TAVR. Paradoxically, these patients are generally higher risk and are thus less favorable candidates for open surgery or traditional alternative-access TAVR. With these considerations in mind, transcaval TAVR was developed as a fully percutaneous, non-surgical approach for aortic valve replacement in patients who are poor candidates for traditional alternative-access TAVR. In this manuscript we describe the principles on which transcaval TAVR was developed, the outcomes from the largest trial completed evaluating this technique as well as describing the technique used to perform this procedure in a case-based format.
\end{abstract}

Keywords: Transcatheter aortic valve replacement (TAVR); alternative access; transcaval

Received: 06 February 2018; Accepted: 29 March 2018; Published: 14 May 2018.

doi: 10.21037 /jovs.2018.04.02

View this article at: http://dx.doi.org/10.21037/jovs.2018.04.02

\section{Introduction (Figure 1)}

Transcatheter aortic valve replacement (TAVR) has been developed as a minimally invasive approach to aortic valve replacement in patients who are intermediate or high risk with severe symptomatic aortic stenosis as an alternative to surgical aortic valve replacement. TAVR is an established procedure; over 250,000 procedures have been performed in over 65 countries across the world to date and more than 100,000 procedures have been performed in the US over the past 5 years (2).

The majority of TAVR cases $(>80 \%)$ can be performed from a percutaneous transfemoral approach (3). However, transfemoral access is not an option in all patients due to diseased or small femoral and pelvic arteries making it unsafe to pass the TAVR delivery sheath without high risk of

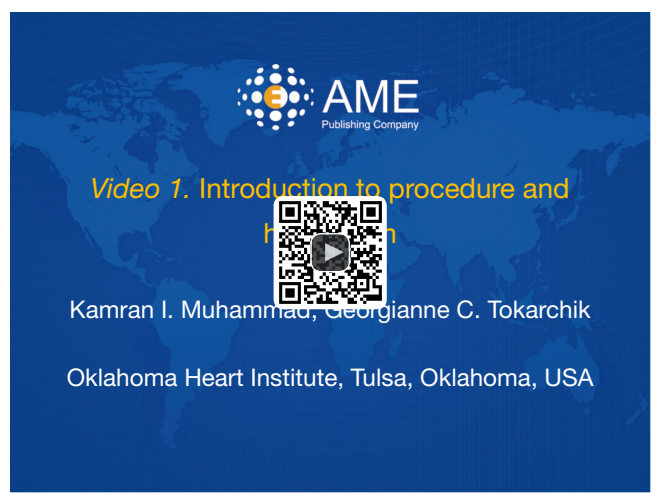

Figure 1 Introduction to procedure and heart team (1). Available online: http://www.asvide.com/article/view/24742 


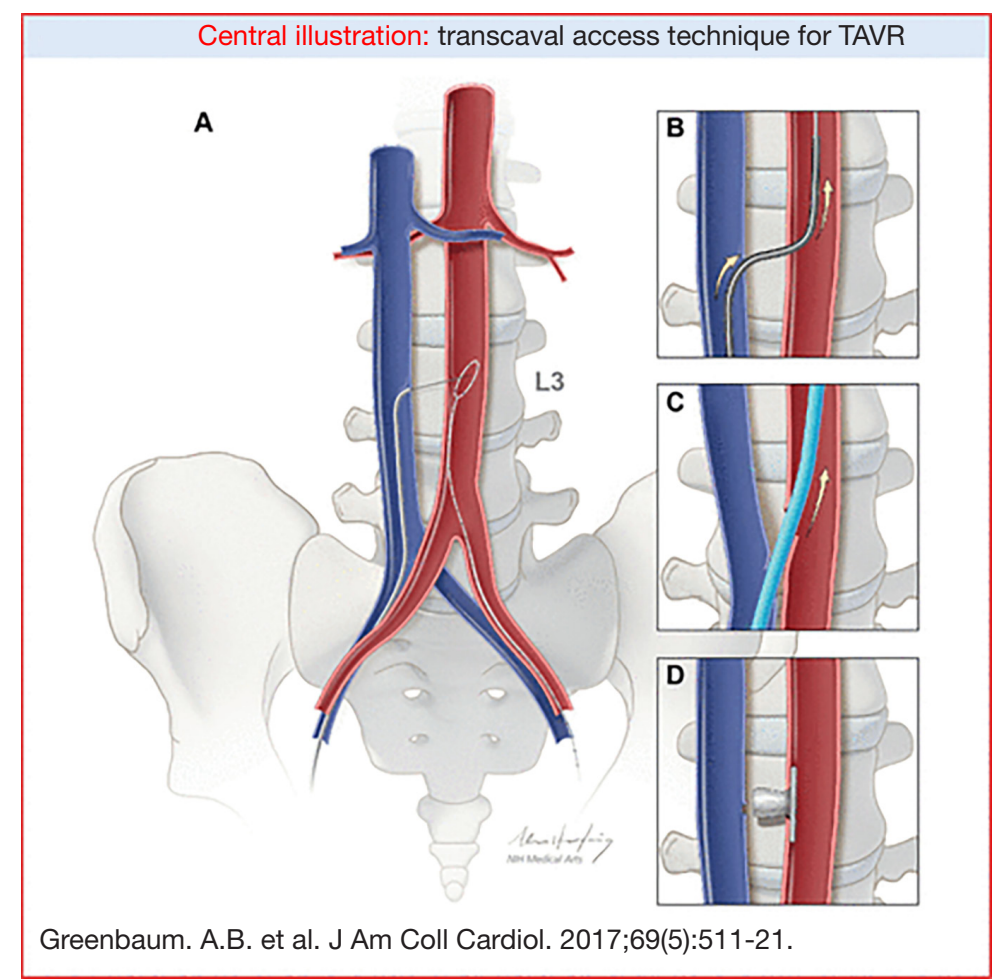

Figure 2 Illustrative transcaval access technique for TAVR. (A) Transcaval access is obtained over an electrified guidewire directed from the inferior vena cava toward a snare in the abdominal aorta; (B) after delivering a microcatheter to exchange for a stiff guidewire; (C) the transcatheter heart valve introducer sheath is advanced from the femoral vein into the abdominal aorta for conventional transfemoral retrograde transcatheter aortic valve replacement (TAVR); (D) the aorto-caval access site is closed with a nitinol cardiac occluder.

injury. This has led to the development of alternative access approaches for delivery of the transcatheter heart valve. Despite the development of a number of alternative access approaches for TAVR, the majority of these approaches are more invasive than transfemoral TAVR and many of them require transthoracic access (transapical/transaortic) which is inferior compared to femoral access (4). This increases the risk for patients compared to transfemoral TAVR which requires no surgery and no instrumentation of the chest cavity. Given the generally high-risk nature of the patients being treated with a non-femoral approach, there has been a great deal of interest in developing an alternative percutaneous non-surgical approach for TAVR. Transcaval TAVR has been developed with these considerations and goals in mind.

\section{Patient selection and workup}

Transcaval access TAVR was developed for patients who are not candidates for traditional transfemoral TAVR due to small pelvic arteries and in whom traditional surgical alternative access was felt to be high-risk. Transcaval access TAVR is a fully percutaneous transfemoral technique that accesses the abdominal aorta through the adjacent inferior vena cava (IVC) which allows for delivery of the transcatheter heart valve from a retrograde approach. This takes advantage of the large and distensible veins in the abdomen and pelvis to deliver the valve to the aorta, thus bypassing the small and diseased arteries of the pelvis. Following completion of the TAVR procedure, the arterial component of the venous-arterial shunt is closed with a nitinol cardiac occluder (Figure 2).

Each patient who is a candidate for TAVR is evaluated with a specialized contrast-enhanced CT scan. When a patient is not a candidate for transfemoral TAVR the preprocedural CT is analyzed for alternative access delivery. In the setting of transcaval TAVR, the CT is evaluated for a calcium-free window on the right wall of the abdominal 


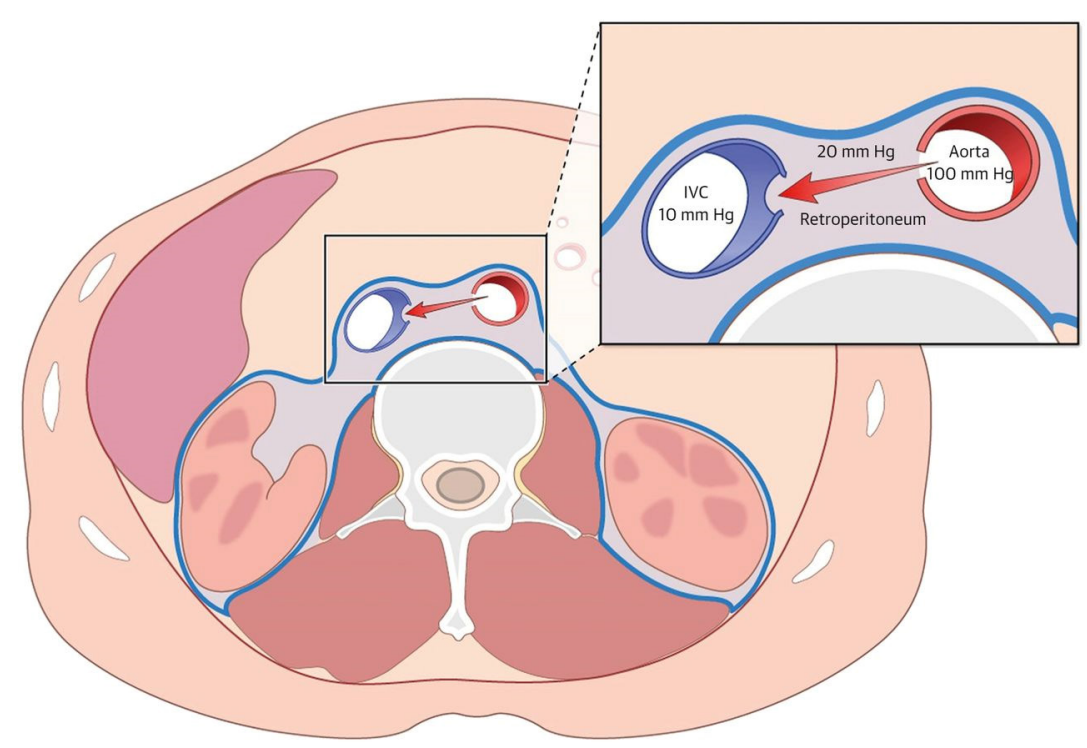

Figure 3 Mechanism of hemodynamic stability after transcaval access. Higher pressure in the relatively confined retroperitoneal space exceeds venous pressure and causes aortic blood to return to the venous circulation through the nearby hole in the inferior vena cava (IVC). The result is an aortocaval fistula rather than hemodynamic collapse.

aorta, close to the vena cava, free of interposed structures where it is feasible to cross from the inferior vena cava to the aorta thus bypassing the iliofemoral vessels (5). In addition, the CT is used to determine bailout with a covered stent in case there is failure of closure with the occluder device.

The physiology of transcaval access provides insight as to why the transcaval access approach is feasible. Studies have demonstrated that the opening created in the vena cava during the procedure serves to decompress aortic bleeding during transcaval access and closure (5). The surrounding retroperitoneal (abdominal) space pressure exceeds the venous pressure and causes blood to return from the aorta into the circulation through the vena cava. The vena cava is the lowest pressure in the abdomen and acts as a natural sink or sump for blood to flow from the aorta to the inferior vena cava (Figure 3).

Initial animal studies performed at the U.S. National Institutes of Health demonstrated animals tolerated the aorto-caval shunt even without closure and that transcaval access was feasible (6). The technique was then performed in humans and again the feasibility and safety of the technique was demonstrated in the first 19 patients who underwent aorto-caval shunt closure with a nitinol occluder (7).

The transcaval approach was subsequently systematically assessed in a multi-center prospective study of 100 patients (8). The study demonstrated that transcaval access was successful in $99 \%$ of the high-risk patients enrolled in the trial (Table 1). Inpatient survival was $96 \%$, and 30 -day survival was $92 \%$ and there were no deaths as a direct result of transcaval access. Second Valve Academic Research Consortium (VARC-2) life-threatening bleeding was $7 \%$ and major vascular complications possibly related to transcaval access were $13 \%$ (Table 2). By comparison, the rates of life-threatening or disabling bleeding for intermediate risk patients in the PARTNER II trial was $22.6 \%$ for transthoracic alternative-access TAVR, and $6.7 \%$ for transfemoral TAVR. Therefore, life-threatening bleeding with transcaval TAVR in high-risk patients in this trial compared favorably with the rates of bleeding in lower risk patients in the PARTNER II intermediate risk trial. In summary, this trial confirmed that transcaval access TAVR was a safe and effective option for high-risk patients with limited options.

\section{Equipment list (Table 3)}

\section{Transcaval case report (Figure 4)}

This is a 75 -year-old frail female with multiple comorbidities including chronic obstructive pulmonary disease, atrial fibrillation, history of cerebrovascular 
Table 1 Baseline characteristics $(\mathrm{N}=100)$

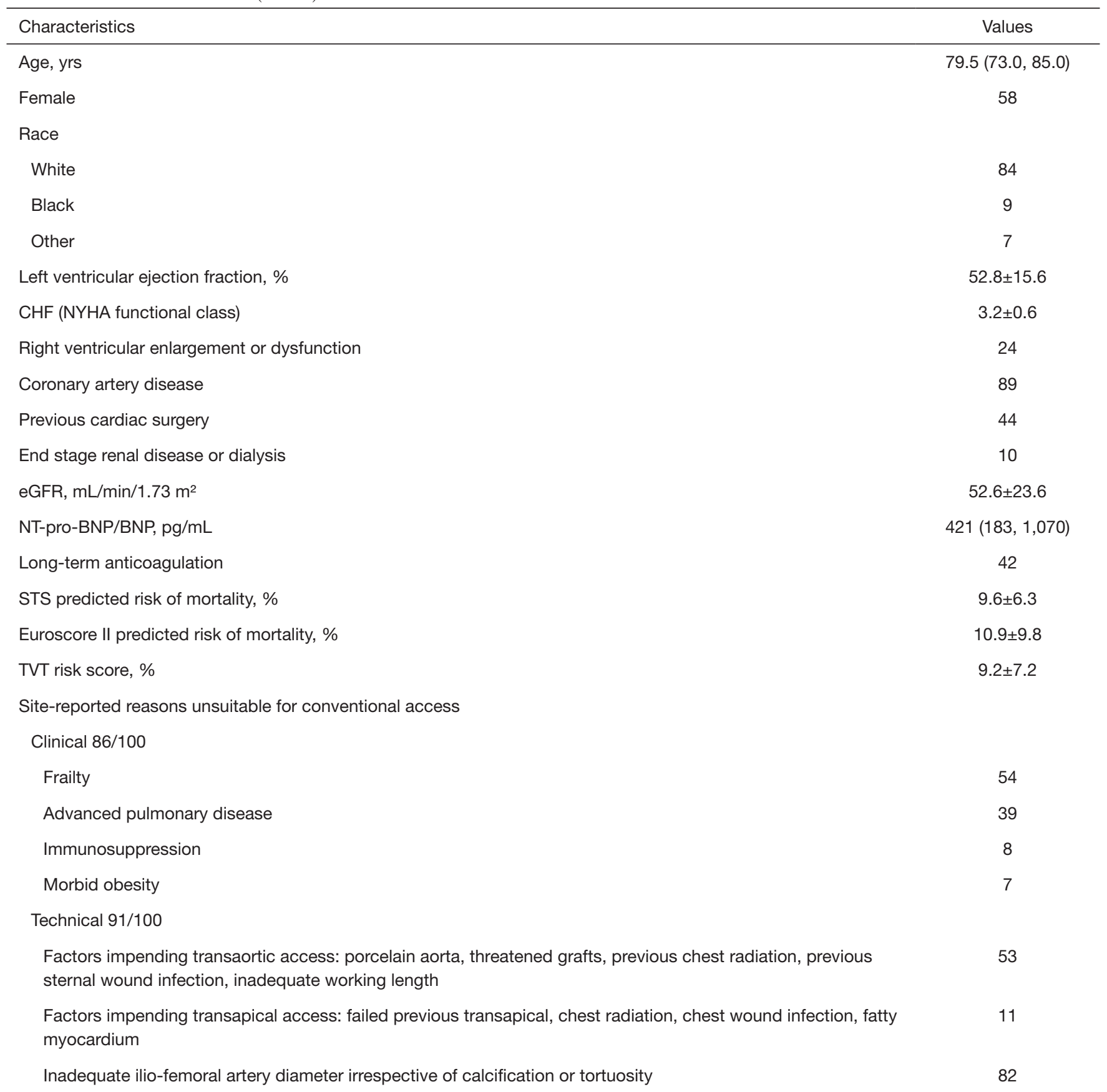

Values are $\mathrm{n}\left(25^{\text {th }}, 75^{\text {th }}\right.$ percentile), $\mathrm{n}$, or mean \pm SD. CHF, congestive heart failure; Egfr, estimated glomerular filtration rate; NYHA, New York Heart Association; NT-proBNP, N-terminal pro-brain natriuretic peptide; STS, Society of Thoracic Surgeons; TVT, Transcatheter Valve Therapy Registry.

accident, coronary artery disease, peripheral arterial disease, severe symptomatic aortic with disabling heart failure symptoms. The patient was evaluated by the cardiac surgery team and felt to be a high risk for surgical aortic valve replacement (30-day STS predicted mortality $8.2 \%$ ).

The patient's procedure was performed in the hybrid cardiac catheterization laboratory at Oklahoma Heart Institute. The transcaval technique has been previously 
Table 2 Outcomes through 30 days $(\mathrm{N}=100)$

\begin{tabular}{|c|c|}
\hline Outcome & Results \\
\hline Death within 30 days & 1 Noncardiovascular \\
\hline Stroke & 5 Ischemic \\
\hline Myocardial infarction & 2 Peri-procedural \\
\hline \multirow[t]{4}{*}{ Acute kidney injury classification } & Grade $0(n=87)$ \\
\hline & Grade $1(n=9)$ \\
\hline & Grade $2(n=0)$ \\
\hline & Grade $3(n=3)$ \\
\hline Transfusion during TAVR/after TAVR/during or after TAVR & $14 / 30 / 35$ \\
\hline Transfusion units among those transfused (median) $(n=35 / 100)$ & $2.0(2.0,4.0)$ \\
\hline Follow-up CT scan before discharge & 87 \\
\hline Post-TAVR LOS (days), median (quartiles) & $4[2-6]$ \\
\hline Post-TAVR ICU LOS (days), median (quartiles) & $1[1-3]$ \\
\hline VARC-2 composite early safety* & 75 \\
\hline
\end{tabular}

*, Second Valve Academic Research Consortium (VARC-2) composite early safety is 30-days freedom from mortality, stroke, life-threatening bleeding, acute kidney injury stage 2 or 3 coronary obstruction requiring intervention, major vascular complication, or valve-related dysfunction requiring repeat procedure. CT, computed tomography; TAVR, transcatheter aortic valve replacement; LOS, length of stay.

described in detail (10). The methodology for interpreting a CT scan for planning transcaval TAVR has also been previously described in detail (5). The standard preprocedural TAVR planning CT scan is analyzed to determine an appropriate preselected target site for crossing from the inferior vena cava into the abdominal aorta (Figure 5A).

Moderate/conscious sedation was administered by anesthesiology. Bilateral percutaneous femoral venous and arterial access was obtained using modified Seldinger technique. Initial simultaneous aortogram and vena cavagram were performed to evaluate the anatomy and correlate with the findings on pre-procedural CT scanning (Figure 5B). A crossing catheter was placed in the inferior vena cava and a goose neck snare at the predetermined crossing site was placed in the aorta. Next an energized 0.014" diameter guidewire was passed from the vena cava across to the aorta and captured by the snare, thereby creating the caval-aortic tract (Figure 5C). Next, through this caval-aortic tract, the transcatheter heart valve sheath (Edwards Lifesciences eSheath $14 \mathrm{~F}$ ) used to deliver the heart valve was then advanced from the IVC into the aorta (Figure 5D). Once in the aorta the patient's iliofemoral arteries that were not adequate for transfemoral access have been bypassed and deployment of a 26-mm Edwards SAPIEN 3 transcatheter heart valve with standard technique was performed from a standard retrograde aortic approach.

After successful placement of the transcatheter aortic valve, the caval-aortic tract was then closed using a nitinol occluder device (10/8 mm Amplatzer Duct Occluder, Abbott). The occluder device is used to close the opening in the side wall of the aorta this is done as the large delivery sheath is removed from the aorta. Closure of the aorta was then evaluated with angiography. Initially angiography demonstrated an aorto-caval shunt and mild retroperitoneal bleeding. Given these findings, we proceeded with adjunctive balloon angioplasty of the infra-renal abdominal 
Table 3 Equipment List

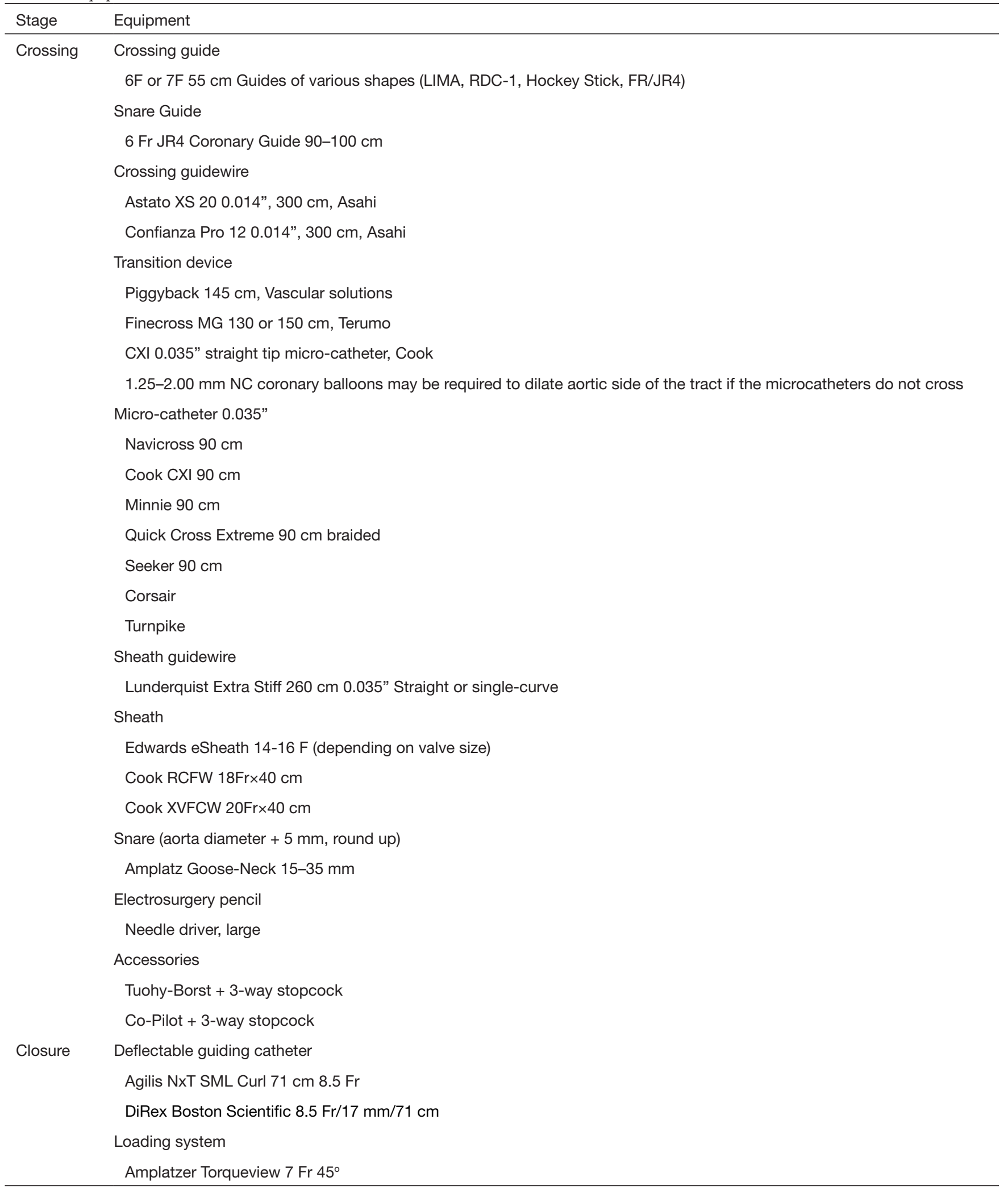

Table 3 (continued) 
Table 3 (continued)

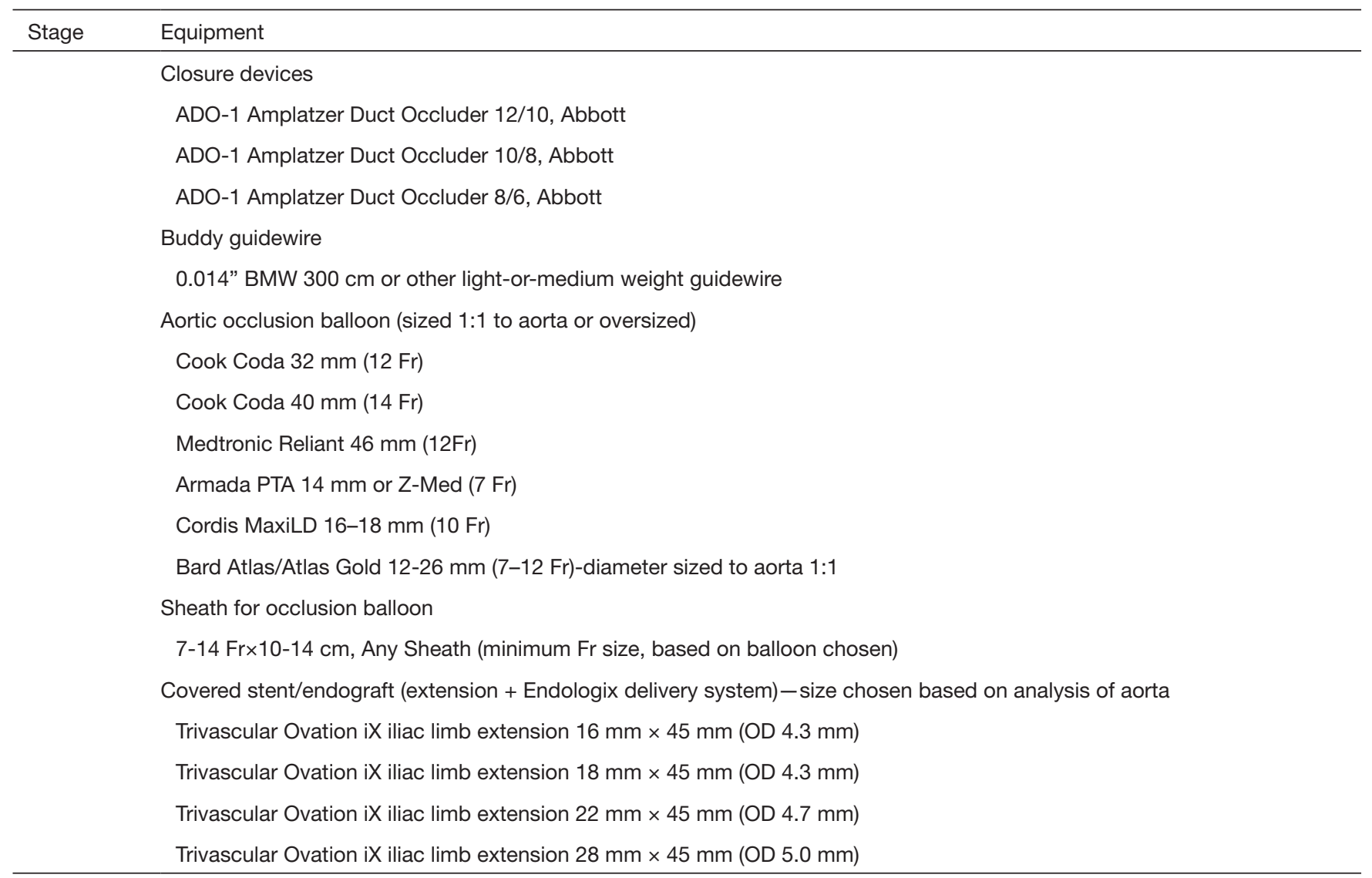

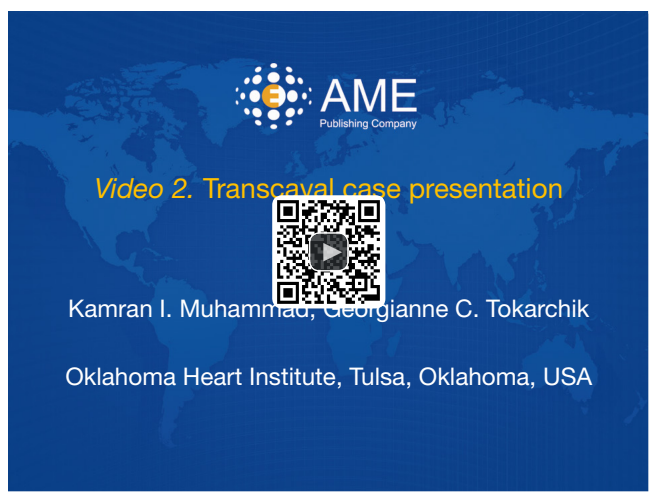

Figure 4 Transcaval case presentation (9).

Available online: http://www.asvide.com/article/view/24743 aorta $(10 \mathrm{~mm} \times 40 \mathrm{~mm}$ Atlas Gold balloon, Bard) simultaneous with re-constraining and slight repositioning of the ADO device, so that it was more perpendicular to the caval-aortic tract with the disc of the device parallel to the aortic wall. It is not uncommon to have a persistent aortocaval shunt immediately post procedure. This is felt to be acceptable unless there is bleeding into the retroperitoneal space or the aorto-caval shunt is felt to be causing heart failure $(7,8)$.

CT of the abdomen and pelvis was performed on postprocedure day 1 and demonstrated no residual aortocaval shunt and no retroperitoneal bleeding (Figure 5E). Study findings demonstrate that $36 \%$ of the aorto-caval fistulas to be closed immediately following transcaval TAVR, 53\% closed by the time of hospital discharge and $64 \%$ of fistulas 

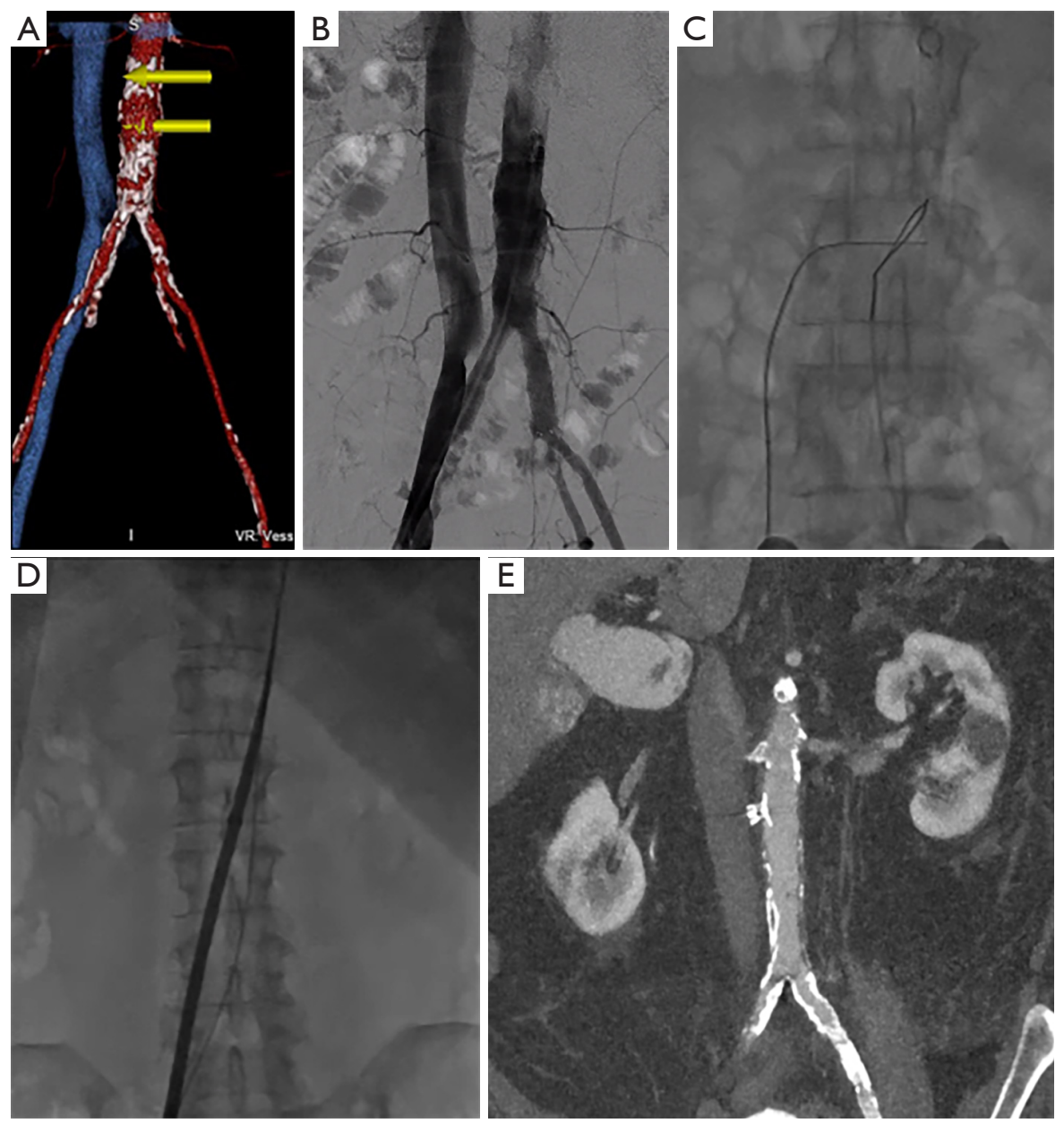

Figure 5 Standard steps for transcaval TAVR procedure. (A) CT plan with yellow arrows showing suitable crossing target; (B) Aortogram/ cavagram; (C) electrified wire crossing into aortic snare; (D) TAVR delivery sheath from femoral vein into aorta; (E) final closure of aortocaval tract with ADO-1 plug.

were closed at 30 days (8).

\section{Post-operative management}

The patient was monitored in the cardiovascular step down unit following the procedure and was out of bed later the same day. The patient did well throughout her hospitalization and was discharged home on day two following his procedure. Follow-up post-procedure CT angiogram of the abdomen and pelvis was performed at 1 month and 1 year following the procedure. Both of these studies demonstrated the closure device to be in good position with no aorto-caval shunting, aortic pseudoaneurysm or other abnormality at the site of the caval-aortic tract. The patient is now greater than one year out following transcaval TAVR and is doing extremely well with no congestive heart failure symptoms with normal function of her aortic valve prosthesis.

\section{Conclusions}

To date, approximately 450 transcaval TAVR procedures have been performed worldwide and this approach is a safe and effective option for patients who are poor candidates for transfemoral TAVR and standard thoracic alternative-access TAVR. As described above, transcaval access and closure for TAVR is standardized and reproducible and overall the approach is safe and effective for high-risk patients with limited options for TAVR. Ongoing follow-up of patients treated with this approach and future studies will help 
us determine the long-term outcomes of this technique, particularly related to transcaval access.

\section{Acknowledgements}

None.

\section{Footnote}

Conflicts of Interest: The authors have no conflicts of interest to declare.

Informed Consent: Written informed consent was obtained from the patient for publication of this manuscript and any accompanying images.

\section{References}

1. Muhammad KI, Tokarchik GC. Introduction to procedure and heart team. Asvide 2018;5:511. Available online: http://www.asvide.com/article/view/24742

2. Vahl TP, Kodali SK, Leon MB. Transcatheter aortic valve replacement 2016: a modern-day "through the lookingglass" adventure. J Am Coll Cardiol 2016;67:1472-87.

3. Holmes DR Jr, Nishimura RA, Grover FL, et al. Annual Outcomes With Transcatheter Valve Therapy: From the STS/ACC TVT Registry. J Am Coll Cardiol

doi: 10.21037 /jovs.2018.04.02

Cite this article as: Muhammad KI, Tokarchik GC. Transcaval transcatheter aortic valve replacement: a visual case review. J Vis Surg 2018;4:102.
2015;66:2813-23.

4. Leon MB, Smith CR, Mack MJ, et al. Transcatheter or Surgical Aortic-Valve Replacement in Intermediate-Risk Patients. N Engl J Med 2016;374:1609-20.

5. Lederman RJ, Greenbaum AB, Rogers T, et al. Anatomic Suitability for Transcaval Access Based on Computed Tomography. JACC Cardiovasc Interv 2017;10:1-10.

6. Halabi M, Ratnayaka K, Faranesh AZ, et al. Aortic access from the vena cava for large caliber transcatheter cardiovascular interventions: pre-clinical validation. J Am Coll Cardiol 2013;61:1745-6.

7. Greenbaum AB, O'Neill WW, Paone G, et al. Caval-aortic access to allow transcatheter aortic valve replacement in otherwise ineligible patients: initial human experience. J Am Coll Cardiol 2014;63:2795-804.

8. Greenbaum AB, Babaliaros VC, Chen MY, et al. Transcaval Access and Closure for Transcatheter Aortic Valve Replacement: A Prospective Investigation. J Am Coll Cardiol 2017;69:511-21.

9. Muhammad KI, Tokarchik GC. Transcaval case presentation. Asvide 2018;5:512. Available online: http:// www.asvide.com/article/view/24743

10. Lederman RJ, Babaliaros VC, Greenbaum AB. How to perform transcaval access and closure for transcatheter aortic valve implantation. Catheter Cardiovasc Interv 2015;86:1242-54. 